\title{
Orientação Social, Papel Sexual e Julgamento Moral: Uma Comparação entre duas Amostras Brasileiras e uma Norueguesa
}

\author{
Angela Biaggio ${ }^{1}$ \\ Universidade Federal do Rio Grande do Sul \\ Arne Vikan \\ Norwegian University for Technology and Natural Science ${ }^{2}$ \\ Cleonice Camino \\ Universidade Federal da Paraíba ${ }^{3}$
}

\begin{abstract}
Resumo
Sessenta estudantes universitários (30 homens e 30 mulheres), de João Pessoa, e 60 estudantes de Porto Alegre, igualmente distribuídos, foram comparados a uma amostra semelhante da Noruega - 120 estudantes universitários ( 60 homens e 60 mulheres). Exceto por uma aparente diferença na orientação cultural entre as mulheres brasileiras, comparações através do teste de moralidade de justiça de Gibbs, do teste ECI da ética do cuidado, do inventário de papéis sexuais de Bem, e do teste de orientação cultural de Triandis mostraram que todas as diferenças foram entre a amostra da Noruega e as amostras do Brasil como um bloco. Os brasileiros estabeleceram uma diferenciação em relação aos papéis sexuais que não foi feita pelos noruegueses, e obtiveram escores mais altos na orientação cultural para o coletivismo. Os noruegueses mostraram mais altos escores no ECI, o que pode ser decorrente de um viés cultural no teste. Não houve diferenças, entre o Brasil e a Noruega, nem na orientação cultural para o individualismo, nem no teste de Gibbs. De uma forma geral, os homens obtiveram escores mais altos na medida do individualismo total e as mulheres no coletivismo vertical. As mulheres de João Pessoa obtiveram escores mais hedonísticos e individualistas do que as mulheres de Porto Alegre, que obtiveram escores mais tradicionais. Palavras-chave: Orientação social; papel sexual; julgamento moral; estudo comparativo.
\end{abstract}

Social Orientation, Sexual Role, and Moral Judgment: A Comparison of two Brazilian and one Norwegian Sample

\begin{abstract}
Thirty female and 30 male university students each from Joao Pessoa and Porto Alegre were compared to a comparable Norwegian sample of 60 female and 60 male students. Except for a suggestion of differences in women's cultural orientation, comparisons on Gibbs' test of justice morality, the ECI test for ethic of care, Bem's sex role inventory, and Triandis' test for cultural orientations showed that all differences were between the Norwegian sample and the Brazilian samples as a unit. Brazilians showed a differentiation of sex roles, which was not shown in Norwegians, and higher scores on the collectivism cultural orientation. Norwegians showed higher scores ECI, which might be because of a culture bias in the test. No difference was shown for individualism cultural orientation, and on Gibbs' test. Men scored higher on the total individualism measure, and women on vertical collectivism. JP women scored as more hedonistic and individual than the PA women, who scores as more traditional than the JP women.

Keywords: Social orientation; sexual role; moral judgment; comparative study.
\end{abstract}

Em termos de estereótipos, a população de João Pessoa (JP) e a de Porto Alegre (PA) são polarizadas cultural e geograficamente. Foi estabelecido que os "gaúchos" são vistos como trabalhadores e individualistas, com uma diferenciação muito clara de papéis sexuais, baseada no machismo; enquanto que os "nordestinos" do litoral são vistos como pouco preocupados com o trabalho, orientados mais para o coletivismo e tendo menor rigidez na diferenciação de papéis sexuais.

Como acontece com todos os estereótipos, estes possuem poucos fundamentos científicos. No entanto, Maia, Gouveia, da Silva Filho, Milfont e Lopes de Andrade (2001) forneceram uma exceção ao apontarem a cultura brasileira como coletivista e a Paraíba como um dos estados mais coletivistas do Brasil (p. 20). Outra exceção pode ser o estudo de Hutz e Koller (1992), que mostra uma diferenciação muito clara de papéis sexuais nos estudantes universitários de Porto Alegre.

\footnotetext{
${ }^{1}$ Este estudo foi iniciado por Angela Biaggio, quando, em 1995, visitou a Noruega como conferencista convidada pela Universidade de Tecnologia e Ciências Naturais da Noruega.

${ }^{2}$ Os autores estão endividados à Prof . Eva Skoe pela condução do estudo na Noruega, e pela organização dos dados.

${ }^{3}$ Endereço para correspondência: Rua da Aurora 201, 908, 58043 270, Miramar, João Pessoa, PB. E-mail:cleocamino@yahoo.com.br
}

O presente estudo, que compara amostras de estudantes de JP e de PA, pode apresentar alguns dados além desses através do uso tanto do teste de Triandis (Gouveia, 1998; Triandis, 1996) para o individualismo-coletivismo, como do teste de Bem para a diferenciação de papéis sexuais (Bem, 1974; Hutz \& Koller, 1992). As expectativas baseadas em estereótipos podem ser as de que os gaúchos, em comparação com os nordestinos, deveriam pontuar mais alto no individualismo e mais baixo no coletivismo, além de apresentar uma diferenciação mais clara nos conceitos sobre os papéis sexuais.

Este estudo também compreende dois testes de raciocínio moral: o teste de Gibbs (Biaggio \& Barreto, 1991; Gibbs, Basinger \& Fuller, 1992), e o teste do cuidado ético de Skoe (Ex.: ver Skoe \& Diessner, 1994). Estes testes referem-se à crítica feita por Gilligan (1982) à teoria do julgamento moral de Kohlberg (1981) Segundo a autora, o conceito de moralidade desenvolvido por Kohlberg pertence a uma moralidade da justiça e dos direitos individuais, dominada pela masculinidade, enquanto que a moralidade característica das mulheres seria focalizada numa ética do cuidado. Isto leva a uma expectativa de que a amostra de JP apresentará escores mais altos na ética do cuidado e mais baixos na moralidade de justiça do que a amostra de PA. O estudo 
incluiu, em adição, um teste de preocupação ambiental (NEP), o qual é relatado em Vikan, Camino e Biaggio (2004).

Um contraste para a comparação das amostras brasileiras foi fornecido pela inclusão de uma amostra de estudantes da Noruega, que tem uma população pequena, homogênea, e que é considerada individualista na orientação social a andrógina em termos dos papéis sexuais. Expectativas referentes aos resultados do estudo podem ser a de que os escores dos noruegueses serão similares aos de PA na medida da cultura e aos de JP nas medidas dos papéis sexuais e do julgamento moral.

\section{Método}

\section{Participantes}

Os participantes foram 30 mulheres e 30 homens de cada uma das duas cidades brasileiras - Porto Alegre (PA) e João Pessoa (JP) - e mais 60 mulheres e 60 homens da cidade de Trondheim, na Noruega (NOR). Seis outras mulheres em João Pessoa foram entrevistadas devido a uma incompreensão dos entrevistadores e foram excluídas da análise. As idades médias das amostras são 22,7 anos, 22,6 anos, e 23,5 anos, respectivamente para JP, PA e NOR. Todos os participantes foram registrados como estudantes, a grande maioria era de estudantes de psicologia e de solteiros (83,3\% e 94,9\% para PA e JP, respectivamente, $93,2 \%$ na NOR); $61,7 \%$ e $53,3 \%$ consideravam-se católicos em PA e JP respectivamente, $10 \%$ e $13,3 \%$ pontuaram a si mesmos como protestantes e $13,3 \%$ e 11,7\% como "espiritualistas"; 79,2\% dos 55,9\% noruegueses que foram perguntados classificaram-se como protestantes e $17,4 \%$ responderam que não tinham nenhuma religião. Uma vez que o protestantismo é a religião oficial da Noruega, com uma participação da população maior do que $90 \%$, pode-se pressupor que os 44,1\% não respondentes tinham também uma filiação religiosa similar à dos respondentes.

\section{Instrumentos}

As medidas foram: o teste de orientação cultural elaborado por Triandis; o teste de papéis sexuais, de Bem; o teste das entrevistas sobre o cuidado ético elaborado por Skoe; o teste para a moralidade de justiça e a escala NEP para a preocupação ambiental. O teste elaborado por Triandis é composto de 32 itens separados em quatro dimensões, cada uma com oito itens: a individualidade vertical (o item 20 tendo sido invertido nas análises), a individualidade horizontal, o coletivismo vertical e o coletivismo horizontal. Cada item é avaliado em uma escala de 9 pontos, que varia de discordo fortemente a concordo fortemente.

Quanto ao teste de Bem (1974), foi utilizada no Brasil uma adaptação da forma original realizada por Oliveira (1972), cuja fidedignidade foi novamente avaliada por Hutz e Koller (1992). Como na forma original, a adaptação contém uma lista de 60 adjetivos, sendo 20 itens neutros, 20 itens que constituem a escala da feminilidade e 20 itens que constituem a escala da masculinidade. A cada item, as pessoas devem responder, em uma escala de sete pontos, o quanto o item se aplica à sua pessoa. Na Noruega, foi utilizada uma forma abreviada que contém 20 itens, sendo 10 para avaliar a masculinidade e 10 para avaliar a feminilidade. Cada item é avaliado em uma escala de cinco pontos.

O teste de moralidade de Gibbs - SROM - é constituído por dois dilemas morais, sendo cada um deles seguido por uma série de questões que operacionalizam os estágios morais - de 1 a 5 - da teoria de Kohlberg de 1976. O teste foi adaptado no Brasil por Biaggio (1989). O escore global desse teste só foi possível de ser calculado para os participantess da Noruega e de JP.

A entrevista do Cuidado Ético de Skoe (ECI) consiste de uma entrevista estruturada, relacionada a um conflito da vida real, gerado pelos participantes, e de três dilemas padronizados: uma gravidez não planejada, uma infidelidade conjugal e o cuidar de um dos pais. As respostas são pontuadas em um dos cinco níveis do cuidado ético.

O NEP avalia o grau de aceitação de uma nova perspectiva ecológica. Este teste consta de 15 itens a respeito dos quais o respondente deve assinalar o seu grau de concordância em uma escala de 5 pontos (Vikan \& cols., 2004).

O ECI e o NEP foram adaptados no Brasil por Angela Biaggio.

\section{Procedimento}

A participação no estudo era voluntária e anônima. Os participantes foram testados primeiro pelo ECI, que requer mais atendimento individual, e em seguida pelo teste de Gibbs. Os outros testes foram então realizados sem uma ordem prefixada. Cada amostra foi entrevistada por dois estudantes supervisionados por um dos autores.

\section{Análises}

Análises estatísticas foram feitas com índices representando a soma dos escores dos itens para cada teste.

\section{Resultados}

O teste central para a presente comparação foi o das orientações culturais de Triandis. Os alfas deste teste foram satisfatórios, sendo que os da amostra brasileira foram um pouco mais elevados do que os da norueguesa. A seguir, serão apresentados os alfas das duas amostras (os alfas da Noruega encontram-se entre parênteses): 0,78 $(0,52), 0,75(0,72), 0,71(0,59)$ e $0,69(0,64)$, respectivamente para o individualismo vertical, o individualismo horizontal, o coletivismo vertical e o coletivismo horizontal. Os desvios-padrão correspondentes foram: 11,$48 ; 10,10 ; 8,53$ e 7,80, com todas as distribuições aproximadamente normais.

As médias para cada orientação do individualismo-coletivismo, como também para as dimensões gerais do individualismocoletivismo, para mulheres e homens em cada amostra, são apresentadas na Tabela 1.

Para efetuar as comparações entre as diferentes amostras com os dados do individualismo-coletivismo foram realizadas várias Análises de Variância com as médias apresentadas na Tabela 1. O resultado da ANOVA para o individualismo total mostra um efeito principal 
Tabela 1

Escores Médios das Orientacões Culturais de Homens e Mulheres de João Pessoa (JP), Porto Alegre (PA) e Noruega (NOR)

\begin{tabular}{lrrrrrrrrr}
\hline & \multicolumn{3}{c}{ João Pessoa } & \multicolumn{3}{c}{ Porto Alegre } & \multicolumn{3}{c}{ Noruega } \\
\hline Orientação cultural & Mulh. & Hom. & Total & Mulh. & Hom. & Total & Mulh. & Hom. & Total \\
\hline Individualismo vertical & 34,07 & 34,86 & 34,47 & 38,03 & 34,40 & 36,21 & 34,44 & 37,80 & 36,16 \\
Individualismo horizontal & 57,00 & 55,37 & 55,08 & 50,63 & 55,67 & 53,15 & 50,07 & 52,95 & 50,76 \\
Coletivismo vertical & 48,20 & 50,93 & 50,16 & 52,87 & 50,07 & 51,46 & 46,35 & 47,07 & 46,68 \\
Coletivismo horizontal & 58,57 & 56,13 & 58,06 & 60,23 & 55,97 & 58,10 & 55,11 & 52,43 & 53,77 \\
Individualismo total & 90,93 & 90,23 & 90,58 & 85,03 & 93,70 & 89,37 & 84,36 & 90,75 & 87,56 \\
Coletivismo total & 106,76 & 106,65 & 106,71 & 113,10 & 106,03 & 109,57 & 101,46 & 99,30 & 100,41 \\
Vertical total & 82,27 & 85,93 & 84,07 & 87,27 & 88,10 & 87,68 & 81,02 & 84,85 & 82,92 \\
Horizontal total & 106,77 & 106,65 & 106,71 & 113,10 & 106,03 & 109,57 & 101,47 & 99,30 & 100,41 \\
\hline
\end{tabular}

Tabela 2

Escores Médios Obtidos no Teste de BEM para Homens e Mulheres de João Pessoa (JP), Porto Alegre (PA) e Noruega (NOR) ${ }^{1}$

\begin{tabular}{lcccccccccc}
\hline & \multicolumn{3}{c}{ João Pessoa } & \multicolumn{3}{c}{ Porto Alegre } & \multicolumn{3}{c}{ Noruega } \\
\hline \multirow{2}{*}{ Masculinidade } & Mulh. & Hom. & Total & Mulh. & Hom. & Total & Mulh. & Hom. \\
Feminilidade & 87,20 & 89,72 & 88,44 & 85,77 & 90,20 & 87,98 & 34,48 & 33,51 & 33,98 & 36 \\
& 91,29 & 86,10 & 88,56 & 91,67 & 87,27 & 89,47 & 37,55 & 35,28 & 36,41 & \\
\hline
\end{tabular}

Nota. ${ }^{1}$ Os escores da amostra brasileira são relacionadas ao BEM completo, enquanto que os escores dos noruegueses se relacionam à versão abreviada.

marginal do sexo, $F(1,230)=4,11 ; p=0,044$, indicando que os homens obtiveram escores mais elevados do que as mulheres. Este efeito, entretanto, não foi confirmado por uma análise separada do individualismo vertical e horizontal. Uma outra ANOVA mostra que no individualismo horizontal houve um efeito principal do país, $F(2,234)=3,24 ; p=0,041$, indicando que os brasileiros obtiveram escores mais elevados do que os noruegueses. Um teste tpara amostras independentes indica que este efeito foi devido, sobretudo, aos escores mais altos das mulheres de João Pessoa do que das mulheres norueguesas, $t(85)=-0,351 ; p<0,01$. As mulheres de João Pessoa também mostraram escores significativamente mais altos do que as mulheres de PA $-t(56)=-2,89 ; p<0,01$. Observa-se que esta foi a única diferença interestadual brasileira nas dimensões do individualismo.

As diferenças mais robustas referem-se à dimensão do coletivismo. Os resultados da ANOVA sobre o coletivismo total mostraram um efeito principal de país, $F(2,230)=10,16$; $p<0,01$, indicando (ver dados da Tabela 1) que os brasileiros obtiveram escores mais altos do que os noruegueses. $\mathrm{O}$ efeito principal de país também ocorreu nas dimensões do coletivismo vertical, $F(2,232)=7,042 ; p<0,01$, e do coletivismo horizontal, $F(2,232)=8,51$; $p<0,01$. Em ambas as dimensões, os estudantes brasileiros pontuaram mais alto do que os estudantes noruegueses. Além disso, observou-se no coletivismo horizontal um efeito principal do sexo, $F(1,232)=9,48 ; p<0,01$, o que significa que as mulheres, obtiveram médias mais elevadas do que os homens. Já no coletivismo vertical, observou-se, de forma significativa $t(58)=2,14 ; p=0,037$, que as mulheres de PA obtiveram média mais elevada do que as de JP. Este efeito relaciona-se à única diferença intra-Brasil observada nas dimensões do coletivismo.

Uma ANOVA comparando a amostra norueguesa com as amostras brasileiras combinadas mostrou a influência de país tanto no índice do coletivismo total como no horizontal, ambos os resultados foram $F(1,232)=18,86 ; p<0,01$ relacionados aos escores mais altos para os brasileiros, como um efeito do sexo no índice do individualismo total $F(1,232)=5,47 ; p=0,02$, que está relacionado aos escores mais altos para os homens (ver'Tabela 1). A diferença entre as médias das diferentes dimensões (ver Tabela 1) foi examinada através do teste $t$ para medidas emparelhadas. Nos resultados, observou-se que os escores do coletivismo total foram significantemente mais elevados do que os escores do individualismo $t(231)=-11,54 ; p<0,01$. Os resultados do teste $t$ referentes às comparações entre as dimensões únicas foram todos significativos $(p<0,01)$, sendo que a menor diferença foi entre as duas dimensões horizontais, $t(234)=-2,86$.

Testes t para medidas emparelhadas também foram feitos para comparar as médias das dimensões em cada amostra. Os resultados para todas as comparações foram significativos $(p<0,01)$, exceto para a diferença entre as dimensões horizontais em todas as amostras e para a diferença entre o individualismo horizontal e o coletivismo vertical na amostra de PA. Estes resultados corroboram as diferenças sugeridas pelas médias da Tabela 1, indicando que o individualismo vertical apresenta, de forma consistente, escores mais baixos do que as outras dimensões. Os maiores escores foram suscitados pelas dimensões horizontais, sendo os do coletivismo horizontal os mais altos.

Os coeficientes de correlação entre as diferentes dimensões do individualismo-coletivismo foram semelhantes nas amostras dos dois países: entre os escores do coletivismo vertical e do coletivismo horizontal foram 0,46 (Noruega) e 0,52 (Brasil); entre o individualismo vertical e o individualismo horizontal foram 0,26 (Noruega) e 0,30 (Brasil); entre o individualismo vertical e o coletivismo vertical foram 0,25 (Noruega) e 0,19 (Brasil); entre o individualismo horizontal e o coletivismo horizontal foram 0,07 (Noruega) e 0,21 (Brasil); entre o individualismo total e o coletivismo total foram 0,13 (Noruega) e - 0,05 (Brasil). 
Tabela 3

Escores Médios do ECI para Mulheres e Homens de João Pessoa (JP), Porto Alegre (PA) e Noruega (NOR)

\begin{tabular}{ccccccccc}
\hline \multicolumn{3}{c}{ Porto Alegre } & \multicolumn{3}{c}{ João Pessoa } & \multicolumn{3}{c}{ Noruega } \\
\hline Mulheres & Homens & Total & Mulheres & Homens & Total & Mulheres & Homens & Total \\
7,55 & 7,93 & 7,74 & 8,00 & 7,84 & 7,92 & 9,55 & 9,21 & 9,38 \\
\hline
\end{tabular}

Em relação às análises do teste de Bem (Tabela 2), uma comparação direta pôde ser feita apenas com as amostras brasileiras, nas quais foram usadas a mesma versão do Bem.

A Tabela 2 sugere uma clara diferença entre o Brasil e a Noruega em relação às escalas da feminilidade e da masculinidade. Enquanto nas amostras brasileiras as mulheres apresentaram escores mais altos do que os homens no índice de feminilidade e os homens apresentaram escores mais altos do que as mulheres no índice de masculinidade, na amostra norueguesa, tanto as mulheres quanto os homens apresentaram escores mais altos no índice de feminilidade. Isto significa que, enquanto os brasileiros parecem se conformar às diferenças tradicionais nos escores dos papéis sexuais, os noruegueses parecem demonstrar uma aceitação geral dos valores femininos.

As diferenças nos escores para a masculinidade e a feminilidade para as mulheres e para os homens, em cada amostra, foram confirmadas pelo teste de Wilcoxon. Os resultados desse teste mostraram valores bi-caudais ao nível $p<0,01$, para todas as diferenças esperadas, exceto para os homens noruegueses. Assim, verificou-se que nas duas amostras brasileiras as mulheres obtiveram escores mais elevados na feminilidade do que na masculinidade $z=-2,21 ; p=0,027$ para JP e $z=-3,95 ; p<0,01$ para a amostra de PA; enquanto os escores obtidos pelos homens foram mais elevados na escala da masculinidade do que na da feminilidade $z=-2,22 ; p=0,026$ e $z=-1,25 ; p=0,024$, para $\mathrm{JP}$ e PA, respectivamente. Na amostra da Noruega, tanto os homens quanto as mulheres apresentaram na feminilidade escores mais elevados do que na masculinidade $2=-3,36 ; p<0,01$ para as mulheres e $z=-1,96 ; p=0,05$ para os homens. Deve-se ressaltar que os noruegueses de ambos os sexos, na maioria dos postos, tiveram escores mais altos para a feminilidade do que para a masculinidade, o que quer dizer que os homens noruegueses demonstraram um viés de resposta significativo na direção da feminilidade.

As correlações entre as escalas de feminilidade e masculinidade foram 0,43 em JP, 0,63 em PA e 0,074 na Noruega. Os alfas foram bastante similares nas amostras brasileiras, os alfas médios foram 0,53 para o índice de feminilidade e 0,56 para o de masculinidade. Os alfas da Noruega foram 0,51 e 0,77 , para feminilidade e masculinidade, respectivamente. As distribuições para as amostras brasileiras foram semelhantes e caracterizadas por picos de dois a cinco, enquanto que as distribuições norueguesas chegaram mais perto de uma distribuição normal.

Os alfas para o ECI foram 0,$73 ; 0,58$ e 0,88 para as amostras de JP, PA e da Noruega, respectivamente. Os desvios-padrão correspondentes foram: 1,64; 1,51 e 1,49 para JP, PA e Noruega.
Também foram realizados cálculos com os escores médios do ECI, os quais são apresentados na Tabela 3.

A Tabela 3 sugere uma diferença entre médias, sendo as mais altas para os noruegueses. Isto foi confirmado por uma ANOVA que mostrou um efeito principal de país, $F(2,234)=30,52$; $p<0,001$. Análises separadas para cada um dos quatro dilemas do ECI também indicaram a influência principal de país, sendo o menor $F(2,234)=9,80 ; p<0,01$ para o dilema da gravidez não planejada, indicando que em todas as comparações os noruegueses obtiveram escores mais elevados. Uma análise mais detalhada foi realizada com o teste t para amostras independentes comparando os dados da Noruega com os de JP ou PA para cada um dos quatro dilemas. Todos os resultados revelaram diferenças significativas, exceto na comparação com JP no dilema da vida real. Em todas as quatro ANOVAS para a Noruega $v s$. as amostras brasileiras combinadas ocorreram escores significantemente mais altos para os noruegueses, sendo o menor $F(1,238)=13,22 ; p<0,01$, para o dilema da vida real. Os testes $t$ para verificar diferenças entre as amostras brasileiras apresentaram escores mais altos para JP do que para PA no dilema da vida real, $t=-4,04(118) ; p<0,01$, e escores mais altos para PA do que para JP no dilema da infidelidade conjugal, $t=3,46(118), p<0,01$.

O teste de Gibbs, com uma avaliação geral que só foi realizada para os estudantes de JP e da Noruega, apresentou médias de respectivamente 3,72 e 3,79 , as quais não foram significativamente diferentes. Os alfas do Gibbs foram 0,45 para a Noruega e 0,55 para JP. Os testes de correlação entre as duas medidas do pensamento moral foram 0,23 para a Noruega e 0,02 para JP, nenhum dos dois estatisticamente significativo.

As análises da correlação entre os escores dos diferentes instrumentos demonstraram bem poucas relações. De fato, não foi observada nenhuma correlação significativa entre qualquer um par de medidas para a amostra da Noruega. Na amostra de JP verificouse uma correlação de 0,37 entre as dimensões da masculinidade e da individualidade vertical, e, na amostra de PA, uma correlação de 0,30 entre a dimensão de feminilidade e o coletivismo vertical e de 0,34 entre a feminilidade e o coletivismo horizontal.

\section{Discussão}

O teste para a orientação cultural apresentou escores mais altos para os brasileiros do que para os noruegueses no coletivismo, mas não apresentou as diferenças esperadas entre as amostras brasileiras. Esse teste ainda apresentou, diferentemente do que se esperava, escores mais altos no individualismo total para os homens e escores mais 
altos no coletivismo horizontal para as mulheres. Diferenças entre as amostras brasileiras são sugeridas pelos escores mais altos das mulheres de JP do que das de PA no individualismo horizontal e das mulheres de PA do que das de JP no coletivismo vertical.

Análises dos índices totais demonstraram escores mais altos nas amostras brasileiras combinadas, em comparação com a norueguesa, para o coletivismo total e para o horizontalismo, assim como sugerem escores mais altos no individualismo total para os homens. As amostras de ambos os países apresentaram escores mais altos para a dimensão do coletivismo do que para a do individualismo e escores mais altos para as dimensões horizontais do que para as verticais. $\mathrm{O}$ individualismo vertical provocou tanto os escores mais baixos de individualismo, como os escores mais baixos do verticalismo em ambas as amostras.

Os resultados dos outros testes não apresentaram diferenças entre as amostras brasileiras. Em vez disto, os brasileiros, unanimemente, foram diferentes dos noruegueses, ao apresentarem um escore diferenciado no teste de Bem de papéis sexuais e ao pontuarem mais baixo no ECI de Skoe.

As correlações entre os testes foram, em geral, pequenas. A falta de correlações entre os testes de Gibbs, ECI e os escores no teste de Bem para os papéis sexuais parece ter alguma relevância teórica, porque representa, com uma possível exceção para a correlação entre o Gibbs e o ECI, resultados contrários à suposição de Gilligan (1982) sobre as moralidades referentes aos dois gêneros, isto é, uma moralidade de justiça (o teste de Gibbs) ligada à masculinidade e uma moralidade do cuidado ligada à feminilidade.

As restrições nas conclusões pertencentes aos resultados relacionam-se, primariamente, às variações nos alfas, para as diferentes medidas, e às características das amostras. Os alfas foram satisfatórios para o teste de Triandis e para o ECI, um pouco baixos para o teste de Bem e não satisfatórios para o teste de Gibbs. Algumas das razões para esta variação nos alfas podem ser as de que o número de testes representou demandas grandes demais para a atenção e a concentração e/ou ocupou demasiado tempo dos participantes. Isto pode ser parte da explicação para os alfas nos testes de Bem e de Gibbs, os quais compreendem muitos itens, mas não é toda a explicação, uma vez que o alfa baixo dos noruegueses para a escala de feminilidade pertenceu à versão de 20 itens do teste de Bem. Outras razões podem ser as instruções insuficientes ou o tempo insuficiente para completar as questões.

As características da amostra foram semelhantes para as três amostras do estudo, mas não representativas do público em geral. Pode-se dizer também que os grupos de estudantes são menos representativos da população geral do Brasil do que da Noruega, porque a percentagem de acadêmicos na população geral é menor no Brasil. Como será discutido, é apenas em relação à amostra da Noruega que as características da amostra deste estudo implicam que as conclusões são atualmente hipóteses que deveriam ser testadas em outras partes da população.

Os alfas para as quatro dimensões do teste de Triandis comparamse favoravelmente aos alfas dos sete países em um estudo recente (Kemmelmeier \& cols., 2003), como o faz a falta de correlações entre as dimensões centrais do individualismo-coletivismo, assim como as correlações mais altas entre o coletivismo ao comparar-se às dimensões do individualismo (Soh \& Leong, 2002). Os escores coletivistas mais altos para ambas as amostras brasileiras, que foram observados neste estudo, sustentam as observações do coletivismo como uma característica da cultura brasileira (Ex.: Gouveia, Clemente \& Espinosa, 2003; Maia \& cols., 2001). Os noruegueses também apresentaram um escore mais alto no coletivismo do que no individualismo. Isto não era esperado, mas pode estar em concordância com o ideal igualitário que é proeminente na Noruega, como o é na cultura Sueca que é bastante similar (Triandis, 1995).

A dicotomia individualismo-coletivismo tem sido alvo freqüente de estudos trans-culturais (Exs. de revisões: Earley \& Gibson, 1998; Kagitcibasi, 1997; Triandis, 1996, 2001) e tem sido criticada por ser excessivamente inclusiva e sem claridade conceitual (Ferreira, Assmar \& Souto, 2002; Voronov \& Singer, 2002). O estabelecimento das orientações vertical e horizontal (Maia \& cols., 2001; Schwartz, 1990) serve, no entanto, para representar uma variação diferenciada nas características de cada cultura. Os estudos de Gouveia e colaboradores ligados aos valores em relação a escores de orientação cultural para a cultura brasileira (Gouveia \& cols., 2003; Maia \& cols., 2001) são especialmente pertinentes para o presente estudo. Os valores relacionados ao coletivismo mais alto dos brasileiros podem ser associados, de uma maneira geral, mais aos valores sociais do que aos valores individuais e, especificamente, aos valores afetividade, apoio social, honestidade, ordem social, pertença, religiosidade e tradição (Maia \& cols., 2001).

As diferenças nas orientações relacionadas ao sexo parecem não terem sido notadas em estudos anteriores empregando o teste de Triandis. $\mathrm{O}$ individualismo, que parece caracterizar os escores dos homens no teste, é em geral associado aos valores pessoais, enquanto que o coletivismo horizontal seria mais característico dos escores das mulheres e estaria relacionado ao apoio social, à convivência, à cooperação, às relações igualitárias e à harmonia do endogrupo (Maia \& cols., 2001; Triandis, 1995). Mais ainda, o individualismo horizontal, que se que se percebeu ser característico das mulheres de JP, quando comparadas às mulheres de PA, pode estar relacionado ao hedonismo, à autodireção e à privacidade. De fato, Maia e colaboradores (2001) descobriram que este tipo de individualismo caracteriza pessoas que procuram ter seu próprio espaço, diferenciando-se de outras pessoas e possuindo uma identidade própria (p. 21). Por outro lado, o coletivismo vertical, que foi observado como uma característica das mulheres de PA, parece relacionar-se com obediência e tradição, isto é, com o respeito às hierarquias sociais. Isto significa que as mulheres do nordeste podem ser autônomas, autoconscientes e rebeldes, enquanto que as gaúchas submetem-se às tradições e vêem seus papéis na manutenção de valores tradicionais.

Em relação ao teste de diferenciação de papéis sexuais, os estudantes de ambas as amostras brasileiras apresentaram, de forma semelhante, escores nas dimensões da feminilidade-masculinidade que estavam de acordo com as diferenças tradicionais. Os escores dos noruegueses, de ambos os sexos, revelaram uma preferência por 
valores femininos. Isto pode, em parte, ser explicado por atos determinados e concretos na política da Noruega (Ex.: pelo menos $40 \%$ dos ministros devem ser mulheres), pela legislação e pela educação que tornam iguais os salários e as oportunidades para os sexos e, em parte, pelo fato de que os homens na amostra eram estudantes de psicologia, os quais são tradicionalmente proponentes dos valores do feminismo soft.

Os brasileiros também demonstraram as mesmas respostas às entrevistas sobre o cuidado ético, as quais levaram a escores mais altos para as respostas norueguesas. As diferenças nos escores não podem ser concebidas como mostrando um cuidado ético mais alto para os noruegueses, pois as respostas foram pontuadas de acordo com um sistema de pontuação que foi estabelecido em relação a um tipo de cultura individualista (EUA). Não fica claro em que extensão isto pode ser transferido como medida das respostas a um tipo de cultura coletivista tal como o Brasil.

Todas as principais diferenças entre os testes apresentados neste artigo foram referentes à comparação entre as amostras brasileiras de um lado e as norueguesas de outro. Pode-se ainda acrescentar que os resultados de um teste adicional sobre a preocupação com o meio ambiente - NEP - indicaram que os brasileiros obtiveram escores similares entre eles e mais altos do que os noruegueses (Vikan \& cols., 2004). Uma conclusão a este respeito pode ser a de que as duas amostras brasileiras, separadas por $3925 \mathrm{~km}$, são partes de uma mesma cultura.

\section{Referências}

Bem, S. L. (1974). The measurement of psychological androgyny. Journal of Consulting and Clinical Psychology, 42, 155-162.

Biaggio, A. (1989, junho). Adaptação brasileira de medida objetiva de reflexão socio-moral. Trabalho apresentado no Congresso Interamericano de Psicologia, Buenos Aires, Argentina.

Biaggio, A. \& Barreto, M. S. L. (1991). Adaptação brasileira de uma medida objetiva de julgamento moral. Arquivos Brasileiros de Psicologia, 43, 107-119.

Earley, P. C. \& Gibson, C. B. (1998). Taking stock in our progress on individualismcollectivism: 100 years of solidarity and community. Journal of Mangement, 24, $1-25$.

Ferreira, M. C., Assmar, E. M. L. \& Souto, S. de O. (2002). O individualismo e o coletivismo como indicadores de culturas nacionais: Convergências e divergências teórico-metodológicas. Psicologia em Estudo, 7, 81-89.
Gibbs, J. C., Basinger, K. S. \& Fuller, D. (1992). Moral maturity: Measuring the development of sosiomoral reflection. New Jersey: Lawrence Erlbaum.

Gilligan, C. (1982). In a different voice: Psychological theory and women's development. Cambridge: Harvard University Press.

Gouveia, V. V., Clemente, M. \& Espinosa, P. (2003). The horizontal and vertical attributes of individualism and collectivism in a Spanish population. The Journal of Social Psychology, 14, 43-63.

Gouveia, V. V., Andrade, J. M., Milfont, T. L., Queiroga, F. \& Dos Santos, W. S. (2003). Dimensões normativas do individualismo e coletivismo: É suficiente a dicotomia pessoal vs. social? Psicologia: Reflexão e Critica, 16, 223-234.

Hutz, C. S. \& Koller, S. H. (1992). A mensuração do gênero: Uma readaptação do BSRI. Psicologia: Reflexão e Crítica, 5, 15-21.

Kagitcibasi, C. (1997). Individualism and collectivism. Em J. Berry, M.-H. Segall \& C. Kagitcibasi (Orgs.), Handbook of cross-cultural psychology (Vol. 3; pp. 1-49). Boston: Allyn and Bacon.

Kemmelmeier, M., Burnstein, E., Krumov, K., Genkova, P., Kanagawa, C., Hirshberg, M. S., Erb, H-P., Weiczorkowska, G. \& Noels, K. A. (2003). Individualism and collectivism, and authoritarianism in seven societies. Journal of Cross-Cultural Psychology, 34, 304-322.

Kohlberg, L (1981). The meaning and measurement of moral development. Worchester: Clark University Press.

Maia, L., Gouveia, V. V., Da Silva Filho, S. B., Milfont, T. L. \& de Andrade, M. W. C. L. (2001). Prioridades valorativas e individualismo-coletivismo: Padrões de convergência. Psicho, 32, 7-24.

Schwartz, S. H. (1990). Individualism-collectivism: Critique and proposed refinements. Journal of Cross-Cultural Psychology, 21, 139-157.

Skoe, E. E. \& Diessner, R. (1994). Ethic of care, justice, identity, and gender: An extension and replication. Merrill-Palmer Quarterb, 40, 272-289.

Soh, S. \& Leong, F. T. C. L. (2002). Validity of vertical and horizontal individualism and collectivism in Singapore. Journal of Cross-Cultural Psychology, 33, 3-15.

Triandis, H. C. (1995). Individualism and collectivism. Boulder, CO: Westview Press.

Triandis, H. C. (1996). The psychological measurement of cultural syndromes. American Psychologist, 51, 407-415.

Triandis, H. C. (2001). Individualism-collectivism and personality. Journal of Personality, 69, 907-924.

Vikan, A., Camino, C. \& Biaggio, A. (2004). Endorsement of the new ecological paradigm: A comparison of two Brazilian- and one Norwegian sample.(manuscrito nãopublicado)

Voronov, M. \& Singer, J. A. (2002). The myth of individualism-collectivism: A critical review. The Journal of Social Psychology, 142, 461-480.

Recebido: 19/05/2004

Aceite final: 24/08/2004

Sobre os autores

Angela Biaggio era PhD em Psicologia Educacional pela Universidade de Wisconsin. Foi Professora de Graduação e Pós-graduação do Instituto de Psicologia da Universidade Federal do Rio Grande do Sul. Faleceu em 2003.

Arne Vikan é Doutor em psicologia pela Universidade de Oslo, Noruega, e Professor do Instituto de Psicologia, Universidade Noruegesa de Tecnologia e Ciencias Naturais, Trondheim, Noruega.

Cleonice Camino é Doutora em Psicologia pela Université Catholique de Lowvain e Professora aposentada do Departamento de Psicologia da Universidade Federal da Paraíba. 